\title{
Simultaneous Diophantine approximation and VIP systems
}

\author{
by \\ Vitaly Bergelson (Columbus, OH), \\ InGER J. HÅLAND KNUTSON (Kristiansand) and \\ RANDALl MCCutcheon (Memphis, TN)
}

\begin{abstract}
1. Introduction. We begin with a celebrated theorem of H. Weyl ([W1], [W2]).
\end{abstract}

TheOREM W. Let $f(t) \in \mathbb{R}[t]$ be a polynomial having at least one coefficient other than the constant term irrational. Then for any $0 \leq \alpha<\beta \leq 1$ there exists an integer $x$ such that $\alpha<f(x)(\bmod 1)<\beta$.

A simple consequence of Theorem $\mathrm{W}$ is that the set $W_{\alpha, \beta}(f)=\{x \in \mathbb{Z}$ : $\alpha<f(x)(\bmod 1)<\beta\}$ is not merely non-empty, but infinite. As a matter of fact, Weyl obtained Theorem $\mathrm{W}$ as a corollary to a limiting theorem which says that the sequence $(f(n))_{n=1}^{\infty}$ is equidistributed mod 1 , which in particular implies that the density of $W_{\alpha, \beta}(f)$, defined to be

$$
d\left(W_{\alpha, \beta}\right)=\lim _{N \rightarrow \infty} \frac{|\{x \in \mathbb{Z}:-N \leq x \leq N, f(x)(\bmod 1) \in(\alpha, \beta)\}|}{2 N+1},
$$

exists and equals $\beta-\alpha$.

We are concerned here with several increasingly powerful notions of largeness pertaining to subsets of $\mathbb{Z}^{d}$. Notice for example that, since $\beta-\alpha>0$, Weyl's theorem implies that $W_{\alpha, \beta}$ is "large" in the sense of having positive density. As a matter of fact, for our special purposes a weaker version of Weyl's theorem, stating only that $W_{\alpha, \beta}$ has positive lower density

$$
\underline{d}\left(W_{\alpha, \beta}\right)=\liminf _{N \rightarrow \infty} \frac{|\{x \in \mathbb{Z}:-N \leq x \leq N, f(x)(\bmod 1) \in(\alpha, \beta)\}|}{2 N+1},
$$

would be a more apt point of departure, in that it may be inferred from

2000 Mathematics Subject Classification: Primary 11J25; Secondary 11J71.

The first and third authors were supported by NSF grants DMS-0345350 and DMS0200700, respectively. 
the other results we discuss. Indeed, the property of having positive lower density is perhaps a more natural notion of largeness than that of having positive (existing) density, owing to its closure under supersets.

The first extension of the weak form of Weyl's equidistribution theorem quoted above that we mention is due to van der Corput ([vdC]); its formulation requires a different and more powerful notion of largeness. A set $S$ in $\mathbb{Z}^{d}$ is syndetic if the union of finitely many of its additive shifts is all of $\mathbb{Z}^{d}$. Alternatively, $S$ is syndetic if it intersects non-trivially any large enough $d$-dimensional cube; namely, if there exists $k$ such that for all choices of $M_{1}, \ldots, M_{d}, S \cap \prod_{i=1}^{d}\left[M_{i}, M_{i}+k\right] \neq \emptyset$. In $\mathbb{Z}$, then, $S$ is syndetic if it intersects non-trivially any large enough interval, i.e. has bounded gaps.

TheOREM vdC. Let $\alpha_{1}, \ldots, \alpha_{n}, \beta_{1}, \ldots, \beta_{n}$ be real numbers and let $m \in \mathbb{N}$. For each $k=1, \ldots, n$, let $f_{k}$ be a real polynomial of $m+k-1$ unknowns. If the system

$$
\begin{aligned}
\alpha_{1} & <f_{1}\left(x_{1}, \ldots, x_{m}\right)-y_{1}<\beta_{1}, \\
\alpha_{2} & <f_{2}\left(x_{1}, \ldots, x_{m}, y_{1}\right)-y_{2}<\beta_{2}, \\
\alpha_{3} & <f_{3}\left(x_{1}, \ldots, x_{m}, y_{1}, y_{2}\right)-y_{3}<\beta_{3}, \\
& \vdots \\
\alpha_{n} & <f_{n}\left(x_{1}, \ldots, x_{m}, y_{1}, \ldots, y_{n-1}\right)-y_{n}<\beta_{n}
\end{aligned}
$$

has at least one integer-valued solution then it has infinitely many integervalued solutions. Moreover, the set of $x=\left(x_{1}, \ldots, x_{m}\right) \in \mathbb{Z}^{m}$ for which there is some $y=\left(y_{1}, \ldots, y_{n}\right), y_{i} \in \mathbb{Z}$, so that $(x, y)$ satisfies the system (1.1) is syndetic.

In light of Theorem $\mathrm{W}$, taking $n=m=1$ in Theorem vdC yields that $W_{\alpha, \beta}(f)$ is in fact syndetic.

Note. Syndeticity of $W_{\alpha, \beta}$ also follows from well-distribution of the sequence $(f(x))_{x \in \mathbb{N}}$, a concept introduced by E. Hlawka [Hl] and G. Petersen $[\mathrm{P}]$ in the mid-fifties. (See also $[\mathrm{F}]$, where well-distribution of the sequence $(f(x))_{x \in \mathbb{N}}$ is established via ergodic considerations.)

The goal of this paper is to strengthen Theorem vdC in two respects. First we shall show that, in the case that the set of solutions of system (1.1) is non-empty, it is large in a sense still more powerful than that of syndeticity. In doing so, we shall be at the same time extending the following result of Furstenberg and Weiss from $[\mathrm{FW}]$.

Theorem FW. Let $\varepsilon>0$ and for each $k=1, \ldots, n$, let $f_{k}$ be a real polynomial of $k$ unknowns vanishing at zero. Then the system 


$$
\begin{aligned}
\left|f_{1}(x)-y_{1}\right| & <\varepsilon, \\
\left|f_{2}\left(x, y_{1}\right)-y_{2}\right| & <\varepsilon, \\
\left|f_{3}\left(x, y_{1}, y_{2}\right)-y_{3}\right| & <\varepsilon \\
& \vdots \\
\left|f_{n}\left(x, y_{1}, \ldots, y_{n-1}\right)-y_{n}\right| & <\varepsilon
\end{aligned}
$$

has non-trivial integer solutions. Indeed, the set of $x \in \mathbb{Z}$ for which there is some solution $\left(x, y_{1}, \ldots, y_{n}\right)$ is $\mathrm{IP}^{*}$. (A set which is $\mathrm{IP}^{*}$ is in particular syndetic; see below for the definition of $\mathrm{IP}^{*}$.)

Second, we shall show that our extension holds for a wide class of generalized polynomials, namely mappings $\mathbb{R}^{n} \rightarrow \mathbb{R}$ one constructs from the constants and coordinate maps $\left(x_{1}, \ldots, x_{n}\right) \mapsto x_{i}$ using not only the conventional arithmetic operations of addition and multiplication (as in conventional polynomials), but also the operation of taking the integer part.

In a moment we shall introduce the remaining notions of largeness germane to our paper. First, however, we note that a natural way of defining a notion of largeness, say in $\mathbb{Z}^{n}$, is to introduce a family $\mathcal{S}$ of subsets of $\mathbb{Z}^{n}$ and declare a set $E \subset \mathbb{Z}$ to be an $\mathcal{S}^{*}$ set if for every $S \in \mathcal{S}, E \cap S \neq \emptyset$. For example, if $\mathcal{S}$ consists of the sets $S$ in $\mathbb{Z}$ having upper density 1 then the $\mathcal{S}^{*}$ sets are precisely those of positive lower density. If $\mathcal{T}$ is the family of subsets of $\mathbb{Z}^{n}$ containing arbitrarily large $n$-dimensional cubes (so-called thick sets), then the $\mathcal{T}^{*}$ sets are precisely those that are syndetic. For more discussion and examples of this type, the reader is referred to [F, Section 9.1].

A set $S \subset \mathbb{Z}^{n}$ is called an IP set if it contains the set of finite sums, without repetition, of a fixed sequence. (Some authors define an IP set to simply be a set of finite sums itself; we wish for the IP property to be closed under supersets, however. By "without repetition" we mean here repetition of the indices, not the elements appearing in the sequence. If an element appears multiple times in the sequence, it may appear an equal number of times in a finite sum. In particular, any set containing 0 is an IP set by default. This is in contrast to the situation in the semigroup $\mathbb{N}$, where all IP sets have infinite cardinality.)

Let us call a set $E \subset \mathbb{Z}^{n}$ an IP* set if $E$ intersects every IP set nontrivially. It is not hard to see that any $\mathrm{IP}^{*}$ set is syndetic, as any set containing arbitrarily large $n$-dimensional cubes may easily be shown to contain an IP set. On the other hand, it is easy to see that not every syndetic set is $\mathrm{IP}^{*}$. For example, every $\mathrm{IP}^{*}$ set must contain 0 , which is of course not true of all syndetic sets. Also, if $E \subset \mathbb{Z}$ is $\mathrm{IP}^{*}$ and $n$ is any non-zero integer then $n \mathbb{Z} \cap E$ is again $\mathrm{IP}^{*}$.

However, the real strength of the $\mathrm{IP}^{*}$ property is that it is preserved under finite intersections; if $S_{1}, \ldots, S_{k}$ are IP* sets then $\bigcap_{i=1}^{k} S_{i}$ is IP*. This 
non-trivial fact is a consequence of the following theorem due to Hindman ([Hi]) which plays a prominent role in our paper. (Later, we shall give a different version of Hindman's theorem.)

TheOrem H1. Let $R$ be an IP set, let $k \in \mathbb{N}$ and suppose $R=R_{1} \cup \cdots$ $\cdots \cup R_{k}$. Then some $R_{i}, 1 \leq i \leq k$, is an IP set.

One may easily check via Theorem $\mathrm{H} 1$ that the $\mathrm{IP}^{*}$ property passes to finite intersections. First we note that it suffices to establish this for intersections of two sets. So let $A$ and $B$ be $\mathrm{IP}^{*}$ sets and suppose $A \cap B$ is not IP*. Then there exists a set of finite sums $R$ in the complement of $A \cap B$, whereby $R=(R \backslash A) \cup(R \backslash B)$. It follows by Theorem H1 that either $R \backslash A$ or $R \backslash B$ is an IP set. In either case this contradicts the fact that $A$ and $B$ are $\mathrm{IP}^{*}$.

We shall not, however, content ourselves with the IP* property. An even stronger notion of largeness may be obtained by considering VIP setsvariants of IP sets having a "polynomial" nature (see $[\mathrm{BFM}])$. Let $\mathcal{F}$ denote the family of finite, non-empty subsets of $\mathbb{N}$. If $(G,+)$ is a commutative group, a function $v: \mathcal{F} \rightarrow G$ (we often use "sequential" notation $(v(\alpha))_{\alpha \in \mathcal{F}}$ as well) is called a VIP system if there exists some non-negative integer $d$ such that for any pairwise disjoint $\alpha_{0}, \ldots, \alpha_{d} \in \mathcal{F}$ we have

$$
\sum_{\substack{\left\{\beta_{1}, \ldots, \beta_{t}\right\} \subset\left\{\alpha_{0}, \ldots, \alpha_{d}\right\} \\ \beta_{i} \neq \beta_{j}, 1 \leq i<j \leq t}}(-1)^{t} v\left(\beta_{1} \cup \cdots \cup \beta_{t}\right)=0 .
$$

If $\left(v_{\alpha}\right)_{\alpha \in \mathcal{F}}$ is a VIP system then the least non-negative $d$ for which (1.2) holds is called the degree of the system. Plainly, VIP systems of degree zero are identically zero. VIP systems of degree 1 are also called IP systems, and one may easily show that a set $R$ is an IP set if and only if there exists an IP system $\mathcal{F} \rightarrow R$. Similarly we say that $R$ is a VIP set if there exists a VIP system $\mathcal{F} \rightarrow R$. Accordingly, a set $E$ is VIP* if it intersects every VIP set non-trivially. The distinction between sets and systems here is very simple. IP sets and VIP sets are sets; IP systems and VIP systems are functions from $\mathcal{F}$ to some group.

A different, though equivalent, characterization of VIP systems is often useful. For $d \in \mathbb{N}$, let $\mathcal{F}_{d}$ denote the family of non-empty subsets of $\mathbb{N}$ having cardinality at most $d$.

Proposition 1.1 ([M, Proposition 2.5]). Let $G$ be an additive abelian group and let $d \in \mathbb{N}$. A function $v: \mathcal{F} \rightarrow G$ is a VIP system of degree at most $d$ if and only if there exists a function from $\mathcal{F}_{d}$ to $G$, written $\gamma \mapsto n_{\gamma}$, $\gamma \in \mathcal{F}_{d}$, such that

$$
v(\alpha)=\sum_{\gamma \subset \alpha, \gamma \in \mathcal{F}_{d}} n_{\gamma}
$$

for all $\alpha \in \mathcal{F}$. 
We shall prove the following theorem, which contains Theorems vdC and FW as special cases:

Theorem A. Let $\alpha_{1}, \ldots, \alpha_{n}, \beta_{1}, \ldots, \beta_{n}$ be real numbers and let $m \in \mathbb{N}$. For each $k=1, \ldots, n$, let $p_{k}$ be a polynomial of $m+k-1$ unknowns. If the system

$$
\begin{aligned}
\alpha_{1} & <p_{1}\left(x_{1}, \ldots, x_{m}\right)-x_{m+1}<\beta_{1}, \\
\alpha_{2} & <p_{2}\left(x_{1}, \ldots, x_{m}, x_{m+1}\right)-x_{m+2}<\beta_{2}, \\
\alpha_{3} & <p_{3}\left(x_{1}, \ldots, x_{m}, x_{m+1}, x_{m+2}\right)-x_{m+3}<\beta_{3}, \\
& \vdots \\
& \vdots \\
\alpha_{n} & <p_{n}\left(x_{1}, \ldots, x_{m}, x_{m+1}, \ldots, x_{m+n-1}\right)-x_{m+n}<\beta_{n}
\end{aligned}
$$

has an integer-valued solution $\left(a_{1}, \ldots, a_{m+n}\right)$ then the set

$$
\begin{aligned}
& \left\{\left(s_{1}, \ldots, s_{m}\right):\right. \text { there exists a solution } \\
& \left.\qquad\left(s_{1}+a_{1}, s_{2}+a_{2}, \ldots, s_{m}+a_{m}, s_{m+1}, \ldots, s_{m+n}\right)\right\}
\end{aligned}
$$

is VIP*.

2. Proof of the main theorem. Before proving Theorem A, we introduce some notation as well as a few lemmas. For $\alpha, \beta \in \mathcal{F}$, we write $\alpha<\beta$ if $i<j$ for every $i \in \alpha$ and every $j \in \beta$. If $\left(\alpha_{i}\right)_{i=1}^{\infty} \subset \mathcal{F}$ with $\alpha_{1}<\alpha_{2}<\cdots$, then the subfamily

$$
\mathcal{F}^{(1)}=\left\{\bigcup_{i \in \beta} \alpha_{i}: \beta \in \mathcal{F}\right\}=F U\left(\left(\alpha_{i}\right)_{i=1}^{\infty}\right)
$$

is called an IP-ring (we borrow this term from Furstenberg and Katznelson). Notice that $\left(\mathcal{F}^{(1)}, \cup\right)$ is isomorphic as a semigroup to $(\mathcal{F}, \cup)$ under the bijection $\pi(\beta)=\bigcup_{i \in \beta} \alpha_{i}$, and it is often useful to think of them interchangeably. For example, if $\mathcal{F}^{(1)}$ is an IP-ring, we call $x: \mathcal{F}^{(1)} \rightarrow G$ a VIP system if $x \circ \pi$ is VIP.

Here now, as promised, is the second formulation of Hindman's theorem. (See [HS, Corollary 5.17].)

TheOREM H2. Let $\mathcal{F}^{(1)}$ be an IP-ring. For any finite coloring of $\mathcal{F}^{(1)}$, there exists a monochromatic IP-ring $\mathcal{F}^{(2)} \subset \mathcal{F}^{(1)}$.

Hindman's theorem has important ramifications for a certain mode of convergence along $\mathcal{F}$ we shall define presently. Suppose that $\left(x_{\alpha}\right)_{\alpha \in \mathcal{F}}$ is an

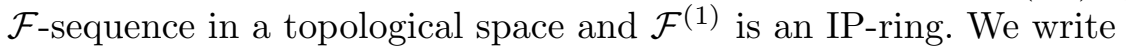

$$
\operatorname{IP}_{\alpha \in \mathcal{F}^{(1)}} x_{\alpha}=z
$$

if for every neighborhood $U$ of $z$ there exists $\beta \in \mathcal{F}$ having the property that $x_{\alpha} \in U$ for every $\alpha \in \mathcal{F}^{(1)}$ with $\alpha>\beta$. 
The following lemma is a simple consequence of Hindman's theorem.

Lemma 2.1. Suppose that $X$ is a compact metric space and $\left(x_{\alpha}\right)_{\alpha \in \mathcal{F}}$ is an $\mathcal{F}$-sequence in $X$. Then for any IP-ring $\mathcal{F}^{(1)}$, there exists an IP-ring $\mathcal{F}^{(2)} \subset \mathcal{F}^{(1)}$ such that

$$
\underset{\alpha \in \mathcal{F}^{(2)}}{\operatorname{IP}-\lim } x_{\alpha}=x
$$

exists.

Proof. Using total boundedness of $X$ and Hindman's theorem, for any IP-ring $\mathcal{F}^{(1)}$ there exists an IP-ring $\mathcal{G} \subset \mathcal{F}^{(1)}$ having the property that the diameter of $\left\{x_{\alpha}: \alpha \in \mathcal{G}\right\}$ is at most $\varepsilon$. Therefore, given $\mathcal{F}^{(1)}$ we may let $\mathcal{F}^{(1)} \supset \mathcal{G}^{(1)} \supset \mathcal{G}^{(2)} \supset \mathcal{G}^{(3)} \supset \cdots$ be a descending sequence of IP-rings such that the diameter of $\left\{x_{\alpha}: \alpha \in \mathcal{G}^{(n)}\right\}$ is at most $1 / n$ for all $n \in \mathbb{N}$. Let now $\alpha_{1}<\alpha_{2}<\cdots$ be an increasing sequence in $\mathcal{F}$ with $\alpha_{i} \in \mathcal{G}^{(i)}, i \in \mathbb{N}$, and let $\mathcal{F}^{(2)}$ consist of the finite unions of this sequence.

In the following lemma and elsewhere in the paper, we denote the fractional part of a real number $x$ by $\{x\}$. That is, $\{x\}=x-[x]$. Depending on the context, we may either take $\{x\}$ to be a real number or an element of the 1 -torus, which we realize as the interval $[0,1)$ under addition modulo 1, equipped with the circle topology, namely that which identifies 0 with 1 . Note in particular that in this topology, $\left\{x_{n}\right\} \rightarrow 0$ if and only if $\left\|x_{n}\right\| \rightarrow 0$, where $\|x\|$ denotes the distance from $x$ to the nearest integer. (In other words, $\|x\|$ is the minimum of $\{x\}$ and $1-\{x\}$. Or, if one prefers, $\|x\|=|x-[x+1 / 2]|$, as $[x+1 / 2]$ is the (possibly not unique) closest integer to $x$.)

Lemma 2.2. Let $v_{i}$ be VIP systems in $\mathbb{R}, i \in \mathbb{N}$. For any IP-ring $\mathcal{F}^{(1)}$ there exists an IP-ring $\mathcal{F}^{(2)}$ such that

$$
\underset{\alpha \in \mathcal{F}^{(2)}}{\operatorname{IP}-\lim }\left\|v_{i}(\alpha)\right\|=0
$$

for all $i \in \mathbb{N}$.

Proof. We prove the result for a single VIP system $v$, whereupon the general result follows by a standard diagonal argument. (In short, one finds a decreasing sequence of IP-rings $\mathcal{G}^{(n)}$ along which the successive VIP systems $v_{n}$ converge to zero, then takes $\mathcal{F}^{(2)}$ to be generated by an increasing sequence $\alpha_{n}$, where $\alpha_{n} \in \mathcal{G}^{(n)}$.) Directly from the definition (or from Proposition 1.1, if one prefers), $\{v\}$ is a VIP system on the 1-torus. Choose $\mathcal{F}^{(2)} \subset \mathcal{F}^{(1)}$ such that

$$
\underset{\alpha \in \mathcal{F}^{(2)}}{\operatorname{IP}-\lim }\{v(\alpha)\}=v_{0}
$$

exists (in the topology of the torus). Letting now all of the $\alpha_{i}$ 's go to $\infty$ in (1.2), we have $v_{0}=0$. This follows from the simple fact that any non-empty 
finite set has one more non-empty subset of odd cardinality than it has non-empty subsets of even cardinality.

Lemma 2.3. Let $v_{i}$ be VIP systems in $\mathbb{R}, i=1,2$, and let $x, c_{1}, c_{2}$ and $k \in \mathbb{R}$ with $0<k<1$. There exists an $\mathrm{IP}$-ring $\mathcal{F}^{(1)}$ such that the following are VIP systems:

(a) $\left(c_{1} v_{1}(\alpha)+c_{2} v_{2}(\alpha)\right)_{\alpha \in \mathcal{F}}$,

(b) $\left(v_{1}(\alpha)\left(v_{2}(\alpha)+x\right)\right)_{\alpha \in \mathcal{F}}$,

(c) $\left(\left[v_{1}(\alpha)+k\right]\right)_{\alpha \in \mathcal{F}^{(1)}}$.

Proof. We use the characterization from Proposition 1.1. Write

$$
v_{i}(\alpha)=\sum_{\gamma \subset \alpha,|\gamma| \leq d} f_{i}(\gamma), \quad i=1,2
$$

Then

$$
c_{1} v_{1}(\alpha)+c_{2} v_{2}(\alpha)=\sum_{\gamma \subset \alpha, \gamma \in \mathcal{F}_{d}}\left(c_{1} f_{1}(\gamma)+c_{2} f_{2}(\gamma)\right),
$$

which proves (a).

Extending the domain of each $f_{i}$ to $\mathcal{F}_{2 d}$ according to $f_{i}(\gamma)=0$ if $d<$ $|\gamma| \leq 2 d$, one has

$$
v_{1}(\alpha)\left(v_{2}(\alpha)+x\right)=x v_{1}(\alpha)+\sum_{\gamma \subset \alpha, \gamma \in \mathcal{F}_{2 d}}\left(\sum_{\gamma_{1} \cup \gamma_{2}=\gamma} f_{1}\left(\gamma_{1}\right) f_{2}\left(\gamma_{2}\right)\right) .
$$

This proves (b).

For (c), by Lemma 2.2 there exists an IP-ring $\mathcal{F}^{(1)}$ (arising from a sequence $\left.\alpha_{1}<\alpha_{2}<\cdots\right)$ such that $\left\|v_{1}(\alpha)\right\|<\min \{k, 1-k\} / 2^{d+1}$ for all $\alpha \in \mathcal{F}^{(1)}$, where $d$ is the degree of the system $v_{1}$. Then for any $\gamma \in \mathcal{F}$ with $|\gamma|=d+1$

$$
\begin{aligned}
V & =\left|\sum_{\emptyset \neq \beta \subset \gamma}(-1)^{|\beta|}\left[v_{1}\left(\bigcup_{i \in \beta} \alpha_{i}\right)+k\right]\right| \\
& =\left|\sum_{\emptyset \neq \beta \subset \gamma}(-1)^{|\beta|}\left(\left[v_{1}\left(\bigcup_{i \in \beta} \alpha_{i}\right)+k\right]-v_{1}\left(\bigcup_{i \in \beta} \alpha_{i}\right)\right)\right| \\
& \leq \sum_{\emptyset \neq \beta \subset \gamma}\left|\left[v_{1}\left(\bigcup_{i \in \beta} \alpha_{i}\right)+k\right]-v_{1}\left(\bigcup_{i \in \beta} \alpha_{i}\right)\right| \\
& =\sum_{\emptyset \neq \beta \subset \gamma}\left\|v_{1}\left(\bigcup_{i \in \beta} \alpha_{i}\right)\right\|<1 .
\end{aligned}
$$

Therefore, since the quantity $V$ is clearly a non-negative integer, it must be zero and $\left(\left[v_{1}(\alpha)+k\right]\right)_{\alpha \in \mathcal{F}^{(1)}}$ satisfies the definition of VIP system of degree $d$. 
By Lemma 2.3(a), (b) we obtain:

Lemma 2.4. Let $p(x)$ be a polynomial mapping $\mathbb{R}^{l} \rightarrow \mathbb{R}^{t}$ with $p(0)=0$ and suppose $(x(\alpha))_{\alpha \in \mathcal{F}}$ is a VIP system in $\mathbb{R}^{l}$. Then $(p(x(\alpha)))_{\alpha \in \mathcal{F}}$ is a VIP system in $\mathbb{R}^{t}$. In particular, if $p(x)$ is an arbitrary polynomial mapping $\mathbb{R}^{l} \rightarrow \mathbb{R}^{t}$ and $c \in \mathbb{R}^{l}$ is constant then $(p(x(\alpha)+c)-p(c))_{\alpha \in \mathcal{F}}$ is a VIP system in $\mathbb{R}^{t}$.

Proof of Theorem A. Let $\left(a_{1}, \ldots, a_{m+n}\right)$ be any integer-valued solution to (1.3) and let $(x(\alpha))_{\alpha \in \mathcal{F}}$ be any VIP system in $\mathbb{Z}^{m}$. Put

$$
\begin{aligned}
v_{1}(\alpha) & =x(\alpha)+\left(a_{1}, \ldots, a_{m}\right) \in \mathbb{Z}^{m}, \\
u_{2}(\alpha) & =p_{1}\left(v_{1}(\alpha)\right)-p_{1}\left(a_{1}, \ldots, a_{m}\right), \\
v_{2}(\alpha) & =\left(v_{1}(\alpha),\left[u_{2}(\alpha)+1 / 2\right]+a_{m+1}\right) \in \mathbb{Z}^{m+1}, \\
& \vdots \\
u_{n+1}(\alpha) & =p_{n}\left(v_{n}(\alpha)\right)-p_{n}\left(a_{1}, \ldots, a_{m+n-1}\right), \\
v_{n+1}(\alpha) & =\left(v_{n}(\alpha),\left[u_{n+1}(\alpha)+1 / 2\right]+a_{m+n}\right) \in \mathbb{Z}^{m+n} .
\end{aligned}
$$

Then by iterated use of Lemmas 2.3(c) and 2.4, there exists an IP-ring $\mathcal{F}^{(1)}$ such that each $\left(v_{k}(\alpha)\right)_{\alpha \in \mathcal{F}^{(1)}}$ is a shift of a VIP system in $\mathbb{Z}^{m+k-1}$ (by $\left(a_{1}, \ldots, a_{m+k-1}\right)$, in fact) and each $\left(u_{k}(\alpha)\right)_{\alpha \in \mathcal{F}(1)}$ is a VIP system in $\mathbb{R}$.

Choose $\varepsilon>0$ so small that

$$
\alpha_{s+1}+\varepsilon<p_{s+1}\left(a_{1}, \ldots, a_{m+s}\right)-a_{m+s+1}<\beta_{s+1}-\varepsilon, \quad s=0,1, \ldots, n-1 .
$$

By Lemma 2.2 there exists $\alpha \in \mathcal{F}^{(1)}$ such that $\left\|u_{i}(\alpha)\right\|<\varepsilon, 2 \leq i \leq n+1$. For this fixed $\alpha$, let $\left(x_{1}, \ldots, x_{m+n}\right)=v_{n+1}(\alpha)$. Then for $1 \leq k \leq n+1$ we have $v_{k}(\alpha)=\left(x_{1}, \ldots, x_{m+k-1}\right)$, and for $1 \leq k \leq n$ we have $x_{m+k}=$ $\left[u_{k+1}(\alpha)+1 / 2\right]+a_{m+k}$ and $u_{k+1}(\alpha)=p_{k}\left(v_{k}(\alpha)\right)-p_{k}\left(a_{1}, \ldots, a_{m+k-1}\right)$, so that

$$
\begin{aligned}
p_{k}\left(x_{1}, \ldots,\right. & \left.x_{m+k-1}\right)-x_{m+k} \\
& =p_{k}\left(v_{k}(\alpha)\right)-x_{m+k} \\
& =u_{k+1}(\alpha)+p_{k}\left(a_{1}, \ldots, a_{m+k-1}\right)-\left[u_{k+1}(\alpha)+1 / 2\right]-a_{m+k} \\
& =p_{k}\left(a_{1}, \ldots, a_{m+k-1}\right)-a_{m+k} \pm\left\|u_{k+1}(\alpha)\right\| .
\end{aligned}
$$

Since $\left\|u_{k+1}(\alpha)\right\|<\varepsilon$, this is enough to show that $\left(x_{1}, \ldots, x_{m+n}\right)$ is a solution to (1.3). Moreover, $\left(x_{1}, \ldots, x_{m}\right)=x(\alpha)+\left(a_{1}, \ldots, a_{m}\right)$, where $(x(\alpha))_{\alpha \in \mathcal{F}}$ was an arbitrary VIP system.

With the aid of Lemma 2.3, one can actually say more. For fixed $l \in \mathbb{N}$, the set of generalized polynomials $\mathbb{R}^{l} \rightarrow \mathbb{R}$ is the smallest set $\mathcal{G}$ that is a function algebra (i.e. is closed under sums and products) containing $\mathbb{R}\left[x_{1}, \ldots, x_{l}\right]$ 
and having the additional property that for all $p \in \mathcal{G}$ the mapping $\mathbf{x} \mapsto[p(\mathbf{x})]$ is in $\mathcal{G}$.

The admissible generalized polynomials $\mathbb{R}^{l} \rightarrow \mathbb{R}$ are defined as the members of the smallest subset $\mathcal{G}_{\mathrm{a}}$ of $\mathcal{G}$ that includes, for $1 \leq i \leq l$, the projections $\left(x_{1}, \ldots, x_{l}\right) \mapsto x_{i}$, is closed under addition, is an ideal in the space of all generalized polynomials, i.e. is such that if $p \in \mathcal{G}_{\mathrm{a}}$ and $q \in \mathcal{G}$ then $p q \in \mathcal{G}_{\mathrm{a}}$, and has the property that for all $m \in \mathbb{N}, c_{1}, \ldots, c_{m} \in \mathbb{R}, p_{1}, \ldots, p_{m} \in \mathcal{G}_{\mathrm{a}}$ and $0<k<1$, the mapping $\mathbf{x} \mapsto\left[\sum_{i=1}^{m} c_{i} p_{i}(\mathbf{x})+k\right]$ is in $\mathcal{G}_{\mathrm{a}}$.

These notions are well defined, however in practice it is somewhat more useful to define a hierarchy whereby new generalized polynomials may be generated from previous ones, as this hierarchy may then be exploited inductively. For example, one might let $G_{0}$ consist of the constant functions $\left(x_{1}, \ldots, x_{l}\right) \mapsto c$, let $G_{1}$ consist of functions of the form $\left(x_{1}, \ldots, x_{l}\right) \mapsto c x_{i}$, then for $n>1$ let $G_{n}$ consist of all functions that can be expressed in the form $g+f, g f$, or $[g]$, where $g, f \in \bigcup_{i=0}^{n-1} G_{i}$. Then

$$
\mathcal{G}=\bigcup_{i=0}^{\infty} G_{i} .
$$

Or, let $H_{1}=G_{1}$ and for $n>1$ let $H_{n}$ consist of those functions $f_{1}+f_{2}$, $f_{1} g$ and $\left[\sum_{i=1}^{m} c_{i} f_{i}+k\right]$ such that $0<k<1, g \in \mathcal{G}, f_{i} \in \bigcup_{i=1}^{n-1} H_{i}, c_{i} \in \mathbb{R}$, $1 \leq i \leq m$. Then

$$
\mathcal{G}_{\mathrm{a}}=\bigcup_{i=1}^{\infty} H_{i} .
$$

Using this characterization of $\mathcal{G}_{\mathrm{a}}$, one may easily establish by induction that if $g(x)$ is admissible then $g(\mathbf{0})=0$. In fact, it is not too difficult to see that usual polynomial mappings $p: \mathbb{R}^{l} \rightarrow \mathbb{R}$ are admissible if and only if $p(\mathbf{0})=0$. The class of admissible generalized polynomials additionally contains such examples as

$$
p\left(x_{1}, x_{2}\right)=\left[\sqrt{3}\left[\sqrt{2} x_{1}^{2} x_{2}\right] x_{2}^{5}+\sqrt{17} x_{1}^{3}+1 / 2\right]\left[\sqrt{5} x_{2}\right] .
$$

Finally, if $t \in \mathbb{N}$ we say a map $p: \mathbb{R}^{l} \rightarrow \mathbb{R}^{t}$ is an admissible generalized polynomial if its coordinate functions are admissible generalized polynomials $\mathbb{R}^{l} \rightarrow \mathbb{R}$. The following more general version of Lemma 2.4 may now be obtained. The routine proof (via Lemma 2.3 and induction) is left to the reader.

LEMma 2.5. Let $p(x)$ be an admissible (respectively non-admissible) generalized polynomial $\mathbb{R}^{l} \rightarrow \mathbb{R}^{t}$ and suppose $(x(\alpha))_{\alpha \in \mathcal{F}}$ is a VIP system in $\mathbb{R}^{l}$. There exists an IP-ring $\mathcal{F}^{(1)}$ such that $(p(x(\alpha)))_{\alpha \in \mathcal{F}^{(1)}}$ is a VIP system (respectively shift of a VIP system) in $\mathbb{R}^{t}$. In particular, if $p(x)$ is a shifted admissible generalized polynomial mapping $\mathbb{R}^{l} \rightarrow \mathbb{R}^{t}$ and $c \in \mathbb{R}^{l}$ is constant 
then there exists an IP-ring $\mathcal{F}^{(1)}$ such that $(p(x(\alpha)+c)-p(c))_{\alpha \in \mathcal{F}^{(1)}}$ is a VIP system in $\mathbb{R}^{t}$.

We remark that by using Lemma 2.5 in place of Lemmas 2.3(c) and 2.4, the following more general form of Theorem A can be obtained by the same method.

TheORem 2.6. Let $\alpha_{1}, \ldots, \alpha_{n}, \beta_{1}, \ldots, \beta_{n}$ be real numbers and let $m \in \mathbb{N}$. For each $k=1, \ldots, n$, let $p_{k}$ be a shifted admissible $\mathbb{R}$-valued generalized polynomial of $m+k-1$ unknowns. If the system

$$
\begin{aligned}
\alpha_{1} & <p_{1}\left(x_{1}, \ldots, x_{m}\right)-x_{m+1}<\beta_{1}, \\
\alpha_{2} & <p_{2}\left(x_{1}, \ldots, x_{m}, x_{m+1}\right)-x_{m+2}<\beta_{2}, \\
\alpha_{3} & <p_{3}\left(x_{1}, \ldots, x_{m}, x_{m+1}, x_{m+2}\right)-x_{m+3}<\beta_{3}, \\
& \vdots \\
\alpha_{n} & <p_{n}\left(x_{1}, \ldots, x_{m}, x_{m+1}, \ldots, x_{m+n-1}\right)-x_{m+n}<\beta_{n}
\end{aligned}
$$

has an integer valued solution $\left(a_{1}, \ldots, a_{m+n}\right)$ then the set

$$
\begin{aligned}
\left\{\left(s_{1}, \ldots, s_{m}\right):\right. \text { there exists a solution } \\
\left.\qquad\left(s_{1}+a_{1}, s_{2}+a_{2}, \ldots, s_{m}+a_{m}, s_{m+1}, \ldots, s_{m+n}\right)\right\}
\end{aligned}
$$

is VIP*.

We remark that Theorem 2.6 does not hold for arbitrary generalized polynomials. For example, if $p(x)=[\pi x] / 2$ then the equation $-1 / 4<$ $p\left(x_{1}\right)-x_{2}<1 / 4$ has the integer-valued solution $(0,0)$. However, one may easily choose an IP system $n$ such that $[\pi n(\alpha)]$ is odd for every $\alpha \in \mathcal{F}$, which implies that

$$
\left\{x_{1}: \exists \text { an integer-valued solution }\left(x_{1}, x_{2}\right)\right\} \cap\{n(\alpha): \alpha \in \mathcal{F}\}=\emptyset .
$$

\section{References}

[BFM] V. Bergelson, H. Furstenberg and R. McCutcheon, IP-sets and polynomial recurrence, Ergodic Theory Dynam. Systems 16 (1996), 963-974.

[vdC] J. G. van der Corput, Diophantische Ungleichungen. II: Rhythmische Systeme. A, B, Acta Math. 59 (1932), 209-328.

[F] H. Furstenberg, Recurrence in Ergodic Theory and Combinatorial Number Theory, M. B. Porter Lectures, Princeton Univ. Press, Princeton, NJ, 1981.

[FW] H. Furstenberg and B. Weiss, Simultaneous Diophantine approximation and IPsets, Acta Arith. 49 (1988), 413-426.

[Hi] N. Hindman, Finite sums from sequences within cells of a partition of N, J. Combin. Theory Ser. A 17 (1974), 1-11.

[HS] N. Hindman and D. Strauss, Algebra in the Stone-Čech Compactification: Theory and Applications, de Gruyter, Berlin, 1998. 
[Hl] E. Hlawka, Zur formalen Theorie der Gleichverteilung in kompakten Gruppen, Rend. Circ. Mat. Palermo (2) 4 (1955), 33-47.

[M] R. McCutcheon, An infinitary polynomial van der Waerden theorem, J. Combin. Theory Ser. A 86 (1999), 214-231.

[P] G. M. Petersen, "Almost convergence" and uniformly distributed sequences, Quart. J. Math. Oxford Ser. (2) 7 (1956), 188-191.

[W1] H. Weyl, Über ein Problem aus dem Gebiete der diophantischen Approximationen, Nachr. Ges. Wiss. Göttingen Math.-Phys. Kl., 1914, 234-244.

[W2] - Ü̈ber die Gleichverteilung von Zahlen mod Eins, Math. Ann. 77 (1916), 313352.

Department of Mathematics

The Ohio State University

Columbus, OH 43210, U.S.A.

E-mail: vitaly@math.ohio-state.edu

Department of Mathematics

Agder University College

N-4604 Kristiansand, Norway

E-mail: Inger.J.Knutson@hia.no
Department of Mathematical Sciences University of Memphis Memphis, TN 38152, U.S.A. E-mail: rmcctchn@memphis.edu

Received on 30.12.2002

and in revised form on 12.7.2004 